\title{
argestre
}

\section{A utilização de recursos audiovisuais no cuidado da criança e acompanhante e sua contribuição na assistência integral}

\author{
The use of audiovisual resources in the care of children and companions and their \\ contribution to comprehensive care
}

\author{
Ana Patrícia da Rocha Lima de Paula1, Kadja Kariny dos Santos Peixoto², Mabelly \\ Cavalcante Rego ${ }^{3}$, Marcela Barbosa de Farias ${ }^{4}$, Ingrid Martins Leite Lúcio ${ }^{5}$
}

\begin{abstract}
Resumo
Introdução e objetivo: A hospitalização e o processo de adoecimento infantil influenciam diretamente o aspecto emocional e de enfrentamento da criança. Neste contexto, destacamos a abordagem à criança e ao acompanhante pelo profissional de enfermagem. Buscou-se relatar as contribuições dos recursos audiovisuais na assistência integral à criança. Método: Trata-se de estudo descritivo, qualitativo, do tipo relato de experiência. $O$ cenário envolve a sala de procedimentos de uma unidade clínica pediátrica de um hospital escola, em Maceió-Alagoas, e as impressões observadas no dia a dia dos profissionais, no primeiro semestre de 2019. Resultados e discussão: Este ambiente terapêutico vem possibilitando o processo de acolhimento e estabelecimento de vínculos. Os recursos audiovisuais permitem a relação das tecnologias duras e leves dentro do ambiente hospitalar através de imagens infantis, musicoterapia, vídeos educativos, desenhos, o lúdico e o brincar de maneira inovadora. Com isso, percebe-se melhor aceitação dos cuidados, a assistência centrada na criança e acompanhante, com uma abordagem humanizada e interdisciplinar. Conclusão: A utilização de recursos audiovisuais na rotina da clínica tem se mostrado positiva, minimizando medos, traumas, tornando as intervenções necessárias mais colaborativas e aproximando o acompanhante, criança e equipe interdisciplinar.
\end{abstract}

Palavras-Chave: Ambiência. Recursos Audiovisuais. Tecnologias leves. Pediatria.

\section{Abstract}

Introduction and objective: Hospitalization and the process of childhood illness directly influence the emotional and coping aspect of the child. In this context we highlight the approach to the child and caregiver by the nursing professional, the ambience and the complementary resources used. We sought to report the contributions of audiovisual

\footnotetext{
1. Especialista. Enfermeira Gerente da Clínica Pediátrica do Hospital Universitário Professor Alberto Antunes - HUPAA. Email: anapatriciapaula1@gmail.com

2. Especialista. Enfermeira Assistencial. Hospital Universitário Professor Alberto Antunes - HUPAA. Email: kadjap4@gmail.com 3.Especialista. Enfermeira Assistencial. Hospital Universitário Professor Alberto Antunes.- HUPAA. Email: mabelly cavalcante@hotmail.com

4 Mestre. Universidade Federal de alagoas - UFAL. Email: marcelinhaa17@hotmail.com

5. Doutora. Universidade Federal de Alagoas - UFAL. Email: ingridmll@esenfar.ufal.br

Correspondência: Hospital Universitário Prof. Alberto Antunes. Av. Lourival Melo Mota. S/N. Tabuleiro dos Martins. S/N. Maceió-Al.
}

Rev. Saúde Digital Tec. Educ., Fortaleza, CE, v. 5, n. 1, p.13-22, jan./abr. 2020.

ISSN: 2525-9563 
resources in comprehensive child care. Method: This is a descriptive, qualitative study of the type experience report. The scenario involves the procedure room of a pediatric clinical unit of a teaching hospital in Maceió-Alagoas and the impressions observed in the professionals' daily routine in the first semester of 2019. Results and discussion: This therapeutic environment has enabled the process welcoming and bonding. The audiovisual resources allow the relationship of hard and light technologies within the hospital environment through children's images, music therapy, educational videos, drawings, the playful and innovative play. Thus, it is perceived better acceptance to care, child-centered care and companion, with a humanized and interdisciplinary approach. Conclusion: The use of audiovisual resources in the clinic routine has been positive, minimizing fears, trauma, making necessary interventions more collaborative and bringing the companion, child and interdisciplinary team closer.

Keywords: Ambience. Audiovisual Resources. Light technologies. Pediatrics.

\section{Introdução}

A hospitalização em consequência da doença modifica consideravelmente a rotina da criança e família e reflete na jornada de adaptação¹. A criança, já física e emocionalmente vulnerável pela enfermidade, enfrenta o afastamento do seu ambiente doméstico e do cotidiano, local onde ocorre o desenvolvimento de seu repertório motor, social, emocional e intelectual ${ }^{2}$, sendo assim, um desafio para a equipe multidisciplinar conciliar essa adaptação à rotina hospitalar e a singularidade de cada família.

A fim de minimizar o sofrimento relacionado ao processo de hospitalização e adoecimento infantil busca-se tornar o ambiente hospitalar menos hostil por meio de um cuidado diferenciado, com foco nas suas necessidades emocionais e sociais, utilizando-se de técnicas que contribuam para uma assistência humanizada ${ }^{3}$.

Desse modo procura-se a valorização da ambiência em saúde que de acordo com a Política Nacional de Humanização compreende o espaço físico entendido como de aspectos social, profissional e de relações interpessoais que proporciona uma atenção acolhedora, resolutiva e humana ${ }^{4}$.

Com vistas a esse propósito, destacam-se as atividades lúdicas no ambiente hospitalar e o brincar com direito da criança, além da maneira como o cuidado multiprofissional é prestado. Os recursos lúdicos mostram-se como um estímulo à diversão, distração e entretenimento e favorecem o desenvolvimento da criança nos aspectos social, psicológico e terapêutico, auxiliam na redução do estresse, medo e ansiedade ${ }^{5}$.

A postura e atuação do profissional de saúde, por meio da assistência integral e humanizada, contribuem favoravelmente para a recuperação da criança na condição de adoecimento, contribuindo para a minimização dos danos causados pelo processo de hospitalização infantil2. 
Neste relato de experiência multiprofissional destaca-se o ambiente da sala de procedimento da clínica pediátrica do hospital escola, atualmente como um espaço que remete ao acolhimento, onde utilizam-se recursos audiovisuais e tecnologias leves nas práticas de cuidado em saúde sistematizadas, individualizada e tendo à criança como foco.

A promoção de um ambiente agradável, com cores suaves e motivos infantis, certamente facilita a interação das crianças com os profissionais e outras crianças, pois um ambiente novo e pessoas estranhas podem gerar medos e apreensão ${ }^{6}$.

A musicoterapia e o uso de vídeos educativos também estão presentes nesse espaço, para auxílio efetivo dos procedimentos, fazendo parte constante da rotina, nos seus variados gostos, idades e particularidades. "A intervenção musical proporciona um cuidado mais humanizado ao paciente, além de ser um recurso facilitador da comunicação"

Outro recurso utilizado nesta sala de procedimentos é a Realidade Virtual mediada pelos óculos em procedimentos dolorosos, este recurso modula a atividade cerebral, modifica a forma como a criança interpreta a dor aguda, substitui o foco de atenção (distração), reduzindo a percepção da criança à dor ${ }^{7}$.

Com o intuito de descrever algumas vivências positivas dessas ações para todos os envolvidos no processo, buscouse relatar as contribuições dos recursos audiovisuais na assistência integral à criança a partir da experiência de profissionais da clínica pediátrica na sala de procedimentos.

\section{Métodos}

Trata-se de estudo descritivo, qualitativo, do tipo relato de experiência, tendo como cenário a unidade clínica pediátrica de um hospital escola, da rede pública de Alagoas, situado na cidade de Maceió. O serviço é referência de internação hospitalar para crianças e adolescentes no estado de Alagoas, principalmente no atendimento às condições crônicas e diagnósticas.

O setor possui capacidade para 24 (vinte e quatro) leitos, sendo organizado em quatro enfermarias, sendo uma para adolescentes, uma para pacientes em pré e pós-operatório e duas para lactentes, pré-escolares e escolares. A sala de procedimento funciona desde outubro de 2018, e antes dela, os procedimentos eram realizados nas enfermarias ou no posto de enfermagem.

Este espaço tem proporcionado um cuidado tranquilo e livre de interrupções. $O$ uso desta sala ocorre nos três turnos, destinado para realização de procedimentos na maioria das vezes de enfermagem, tais como: punção venosa, coleta de exames, curativos, cateterismo vesical, sondagem, realização de avaliação clínica e urgência quando necessário. 
Dentre os profissionais destacam-se aqueles da equipe de enfermagem e médica, além dos fisioterapeutas. Como se trata de hospital escola é comum o envolvimento de graduação e pósgraduação.

À criança e seu acompanhante, são explicados sobre o procedimento que será realizado, sendo então estabelecida a utilização de algumas estratégias que promovam um cuidado diferenciado e menos traumático, ressignificando 0 conceito de sala de procedimento dentro do serviço de saúde.

Neste espaço, a cama fica de frente para o computador, onde o mesmo é utilizado não apenas como armazenamento de informações (Prontuário Eletrônico) do paciente e família, mas vislumbrou-se que poderia ser um recurso audiovisual dando acessibilidade ao desejo da criança. Pergunta-se a ela sobre sua preferência por vídeos e músicas como forma de entretenimento e distração além de incluir o acompanhante nesta escolha.

O desenvolvimento desses procedimentos ocorre com o auxílio de recursos audiovisuais na sala de procedimento que proporciona um ambiente mais tranquilo para o paciente, acompanhante e profissionais, de modo a minimizar $o$ estresse e sentimentos negativos decorrentes da hospitalização, principalmente com procedimentos.

Os procedimentos realizados nesta sala são registrados no prontuário e livro próprio para este fim, com dados técnicos, material, profissionais envolvidos, e abordagem da criança antes, durante e após o procedimento por meio de recursos audiovisuais. Durante os procedimentos as crianças apresentam-se mais receptivas e acolhidas em relação a abordagem da equipe.

Os dados deste relato resultam da prática dos autores, das observações diárias do serviço durante os procedimentos realizados com as crianças assistidas no primeiro semestre de 2019 e do livro de registro do setor.

Neste período ficaram internadas um quantitativo de 247 crianças, e na rotina assistencial foram observadas as mudanças positivas que ocorreram após a implantação deste novo espaço como serão descritas a seguir.

\section{Resultados}

$\mathrm{Na}$ hospitalização, a criança encontra-se fragilizada devido à doença e a tarefa do cuidar não é fácil, por isso tornar o ambiente estimulante e não ameaçador, contribui para que a criança enfrente o processo de adoecimento $\mathrm{e}$ hospitalização de forma construtiva, dinâmica e saudável. Trabalhar com atividades lúdicas é prática inerente ao cuidado pediátrico e deve ser garantido como direito ${ }^{8}$.

A atenção sensível e empática converge para o que é recomendado pela Política Nacional de Humanização do Ministério da Saúde. Nesta perspectiva, o 
uso das tecnologias em saúde está entre as estratégias que tornam possível a criação de um espaço hospitalar mais humanizado 9 .

As tecnologias leves promovem acolhimento, vínculo, autonomização, conforto e os estudos mostram que seu uso interfere na produção do cuidado para concretizar o processo de trabalho.

A utilização dessas tecnologias contempla a existência de um objeto de trabalho dinâmico, em contínuo movimento, não mais estático, passivo ou reduzido a um corpo físico. Esse objeto exige dos profissionais da saúde, especialmente do enfermeiro, uma capacidade diferenciada no olhar a ele concedido a fim de que percebam essa dinamicidade e pluralidade, que desafiam os sujeitos à criatividade, à escuta, à flexibilidade e ao cuidado sensível ${ }^{10}$.

Embora a hospitalização geralmente represente um período estressante para as crianças e famílias, ela também representa uma oportunidade para facilitar uma mudança positiva na própria criança e entre os membros da família ${ }^{11}$.

É imprescindível que a abordagem do profissional ao paciente e acompanhante ocorra ainda no leito, iniciando um diálogo participativo, oportunizando a escuta ativa, estabelecendo vínculos com o intuito de minimizar os medos e traumas decorrentes do processo de hospitalização ${ }^{12}$.

O foco do processo de trabalho vivenciado na sala de procedimentos do local do estudo está centrado no uso de tecnologias leves e audiovisuais, com o intuito de proporcionar privacidade ao paciente e família, humanização do cuidado e também melhores condições de trabalho aos profissionais que fazem parte da equipe.

Neste ambiente é garantido a privacidade e o acolhimento, através de uma sala colorida, silenciosa e calma, onde a sua entrada é apresentada com decoração como motivo infantil, sobressaindo diante dos equipamentos e insumos que são utilizados, oportunizando a criação de um novo conceito de sala dentro do serviço de saúde, como um local de promoção de bem-estar a todos envolvidos e construção de relações subjetivas e transformadoras.

Embora este relato de experiência tenha como objeto retratar as contribuições da utilização dos recursos audiovisuais no cuidado à criança e acompanhante, e não mensurá-las, como vistas ao seu bem estar, conforto e minimização do estresse decorrentes de procedimentos, pode subsidiar no futuro a estruturação de indicadores sociais nesse contexto assistencial, visto que estes possuem um papel importante na discussão política e social e para fins de formulação e reformulação de políticas públicas, em especial voltada à criança.

Uma classificação relevante para a Análise e Formulação de Políticas Sociais é a diferenciação dos Indicadores Sociais quanto a natureza do que é indicado, se recurso (indicador-insumo), realidade 
empírica (indicador-produto) ou processo (indicador processo) ${ }^{13}$.

Os indicadores-insumo (input indicators) correspondem às medidas associadas à disponibilidade de recursos humanos, financeiros ou equipamentos alocados para um processo ou programa que afeta uma das dimensões da realidade social ${ }^{13}$.

$\mathrm{Na}$ realidade descrita nesta experiência, podemos apontar como indicadores os profissionais da equipe multidisciplinar da unidade clínica e os equipamentos e/ou recursos disponíveis para a promoção desse cuidado

As intervenções audiovisuais foram realizadas durante todos os procedimentos de enfermagem executados, desde os acessos venosos periféricos, sondagens vesicais, sondagens nasogástricas, orogástricas e enterais, curativos entre outros.

Em relação ao material audiovisual utilizado, há a participação da criança (do lactente ao adolescente) e do acompanhante; quando a criança é maior perguntamos a sua preferência priorizando desenhos educativos e gostos musicais; Tratando-se de lactentes solicita - se a sugestão do acompanhante; Além disso, chama-se a atenção das crianças em relação ao ambiente lúdico, onde as paredes são ilustradas com desenhos infantis e coloridos.

Em relação à reação das crianças as mesmas apresentam-se mais colaborativas e mais tranquilas na hora dos procedimentos, muitas manifestam 0 desejo de permanecer na sala após o termino das intervenções;

Em alguns contextos, os indicadores de insumo e processo costumam ser chamados de indicadores de esforço, e os indicadores-produto de indicadores de resultados, por se tratarem de desdobramentos mais gerais, a serem alcançados no médio prazo ${ }^{13}$. Um estudo de outra natureza é recomendado para se determinar estes indicadores de modo a quantifica-los.

O que se observa na prática empírica é que tem ocorrido uma transformação no ambiente e em todos os atores envolvidos, permitindo a promoção de um ambiente terapêutico onde o foco é a integralidade do cuidado, proporcionando um cuidado humanizado com a intervenção dos procedimentos de maneira lúdica $\mathrm{e}$ sensível, estabelecendo empatia e consequentemente vínculos profissionaisestudantes-paciente-acompanhante.

Os sentimentos e sensações desagradáveis provocados pela hospitalização infantil geralmente se potencializam quando a equipe de saúde não está preparada para prestar o cuidado de forma humanizada e condizente com o universo da criança ${ }^{14}$.

Especificamente na Enfermagem, o cuidar do outro é a essência dessa dicotomia entre ciência e arte, que são os pilares profissionais. Cuidar é estar aberto ao outro, utilizar-se do conhecimento técnico científico e expressivo, relacionar- 
se com o outro com respeito, com sensibilidade, expressando-se num toque, num olhar, num cantarolar de uma canção ou contando uma história.

A utilização dos recursos audiovisuais nos procedimentos, favorece 0 desenvolvimento da criança no âmbito social, psicológico e terapêutico, auxiliando na diminuição do estresse, medo e ansiedade. Nesta perspectiva, apresenta potencial agregador ao cuidado de enfermagem especialmente na realização de procedimentos dolorosos com a criança.

Os recursos audiovisuais, nesse contexto, revelam-se como estímulo a diversão e ao entretenimento, além de permitir ao profissional aproximar-se e criar um vínculo afetivo.

É considerada uma estratégia de humanização, pois proporciona efeitos benéficos, como a melhoria do quadro clínico devido à interação com os profissionais e a diminuição da ansiedade causada pela longa permanência hospitalar e pelos diversos procedimentos dolorosos.

Ainda existem dificuldades para a implantação dessas práticas no cuidado em saúde, algumas relacionadas à insuficiência de recursos humanos, à falta de materiais, de tempo pelos profissionais, e até mesmo o desconhecimento de como realizá-1o ${ }^{8}$.

Com este estudo observa-se que os resultados positivos na assistência integral a curto prazo são a realização dos procedimentos de forma mais rápida, eficaz e mais tranquila, a prestação de um atendimento individualizado e humanizado; os resultados a médio prazo foram a diminuição dos medos nas próximas internações em relação aos procedimentos e relacionamento com a equipe; os resultados a longo prazo incluem o fortalecimento da equipe com a atuação e envolvimento multiprofissional, a recuperação mais rápida das crianças, pois cooperam na realização dos procedimentos e a mudança de atitude dos profissionais de saúde em reconhecer que o ambiente lúdico favorece o processo de recuperação das crianças.

Encontramo-nos em construção de retrato aproximado de determinadas dimensões da realidade social vivenciada. É necessário que a equipe de enfermagem sistematize os cuidados necessários a cada criança, identificando suas necessidades e capacidades de superar situações de sofrimento e dor com mais benefícios que prejuízos. Atitudes nesse sentido podem transformar situações difíceis em experiências ricas e em momentos que contribuam para a saúde da criança ${ }^{12}$.

Para tanto, reconhece-se que para tornar-se efetiva nas unidades de internamento pediátrico, é necessário a sensibilização dos órgãos gestores, para que promovam, não apenas uma reorganização do trabalho da equipe multiprofissional em saúde, mas, recursos materiais para concretizá-la, promovendo ainda uma capacitação continuada com os 
profissionais atuantes nas unidades de internação infantil ${ }^{15}$.

\section{Conclusão}

A sala de procedimento e a forma como o cuidado é realizado nela, mostra-se como recurso facilitador na realização de procedimentos pediátricos, englobando atividades especializadas e direcionadas por profissionais capacitados para promover o bem-estar físico e emocional da criança ao experimentar uma situação de vida incomum à sua idade, como a hospitalização.

Dessa forma, o auxílio de recursos audiovisuais possibilita tornar menos complexos os procedimentos realizados, proporcionando a empatia entre os profissionais da equipe, o paciente e seu acompanhante, tornando o ambiente de trabalho mais sutil e menos hostil.

O espaço tornou-se mais receptivo e acolhedor por suas características infantis, gerando humanização na assistência, tornando a criança o centro da atenção e promovendo conforto e bem-estar respeitando as suas variadas particularidades.

Espera-se, portanto, que este estudo possa incentivar e estimular a equipe interdisciplinar para promover um novo olhar na assistência a estas crianças e familiares, além de contribuir para minimizar as consequências traumáticas da hospitalização buscando valorizar os efeitos benéficos deste processo.

\section{Referências}

1. Freitas HBM; Voltani SSAA.

Therapeutic play in the pediatric urgent and emergency department: an integrative literature review. Cogitare Enferm, volume 21, n.1, p.01-08, jan- mar.2016.

Disponível em: < file:///C:/Users/win10/Downloads/40728169625-3-PB.pdf>. Acesso em: 10 abr. 2019.

2. Ferreira CCM; Remedi PP; Lima RAGA Música como Recurso no Cuidado à Criança Hospitalizada: Uma Intervenção Possível? Rev. REBEn- Revista Brasileira de Enfermagem, volume 59, n.5, p.692, 2006. Disponível em:

<http://www.scielo.br/pdf/reben/v59n5/v59 n5a18.pdf>. Acesso em: 10 set. 2019.

3. Caleffi CCF et al. Contribuição do brinquedo terapêutico estruturado em um modelo de cuidado de enfermagem para crianças hospitalizadas. Rev Gaúcha Enferm, volume 37, n.2, p. 01-08, 2016. Disponível em: < http://www.scielo.br/pdf/rgenf/v37n2/01026933-rgenf-1983-144720160258131.pdf>. Acesso em: 02 set. 2019.

4. Ministério da Saúde (BR). Secretaria de Atenção à Saúde. Núcleo Técnico da Política de Humanização.

Ambiência.2ª̀ed. Brasília, DF, 2010.

5. Alcântara PL et al. Efeito da interação com palhaços nos sinais vitais e na comunicação não verbal de crianças hospitalizadas. Rev Paulista de Pediatria, volume 34, n. 4, p. 432-438, 2016.

Disponível em:

<https://www.sciencedirect.com/science/ar ticle/pii/S01030582160 00186>. Acesso em: 01 set. 2019.

6. Cruz DSM; Costa SFG; Nóbrega MML; Assistência Humanizada à Criança Hospitalizada. Rev Rene, volume 7, n.3, p., junho, 2006. Disponível em <http://periodicos.ufc.br/rene/article/view/5 447/3962>. Acesso em: 01 de setembro de 2019.

7. Farias MB; Lúcio IML; Vieira ACS, Albuquerque MCS. A experiência da 
realidade virtual com crianças em procedimentos dolorosos. Revista de Saúde Digital e Tecnologias Educacionais, volume 4, n.2, p.1-6. Disponível em: http://periodicos.ufc.br/resdite/index.

Acesso em 02 fev. 2020.

8. Nicola GDO et al. Percepções do familiar cuidador acerca do cuidado lúdico à criança hospitalizada. Rev de enfermagem UFPE, volume 8, n. 4, p. 981986, 2014. Disponível em: < file://C:/Users/win10/Downloads/976918251-1-PB.pdf>. Acesso em: 05 set. 2019.

9. Francischinelli AGB; Almeida FA; Fernandes DMS. Routine use of therapeutic play in the care of hospitalized children: nurses' perceptions. Acta Paul Enferm, volume 25, n.1, p. 1-6, abr. 2012. Disponível em:

<http://www.scielo.br/pdf/ape/v25n1/v25n1 a04 .pdf>. Acesso em: 02 set. 2019.

10. Rossi FR; Lima MADS; Acolhimento: Tecnologias Leves nos Processos Gerenciais do Enfermeiro. Rev. REBEnRevista Brasileira de Enfermagem, volume 58, p.306, 2005. Disponível em: $<$ http://www.scielo.br/pdf/reben/v58n3/a10v 58n3.pdf>. Acesso em: 09 set. 2019.

11. Wong DL. Fundamentos da Enfermagem Pediátrica. 9ํecição. Rio de Janeiro: Elsevier, 2014.

12. Silva DF; Brandão EC. As práticas lúdicas no cotidiano do cuidar em enfermagem pediátrica. Rev de Enfermagem da FACIPLAC, volume 1, $\mathrm{n}$. 1, 2017. Disponível em: <http://www.scielo.br/pdf/ean/v13n4/v13n4 a16>. Acesso em: 05 set. 2019.

13. Carley M. Indicadores sociais: teoria e prática. Rio de Janeiro, Zahar, 1985.

14. Malaquias TSM, et al. O uso do brinquedo durante a hospitalização infantil: saberes e práticas da equipe de enfermagem. Ciência, Cuidado e Saúde, volume 13, n. 1, p. 97-103, 2014.

Disponível em:

<ile://C:/Users/win10/Downloads/2180291143-1-SP.pdf>. Acesso em: 05 set. 2019.

15. Lemos I et al. Brinquedo terapêutico no procedimento de punção venosa: estratégia para reduzir alterações comportamentais. Rev Cuid, volume 7, n.1, p. 1-8, out. 2016. Disponível em: < https://revistacuidarte.udes.edu.co/index.p hp/cuidarte>. Acesso em: 10 set. 2019. 


\section{Como citar este artigo}

Paula APRL de; Peixoto KKS; Rego MC; Farias MB de; Lúcio IML. A utilização de recursos audiovisuais no cuidado da criança e acompanhante e sua contribuição na assistência integral. Revista de Saúde Digital e Tecnologias Educacionais. [online], volume 5, n. 1. Editor responsável: Luiz Roberto de Oliveira. Fortaleza, mês e ano, p. 1322. Disponível em: http://periodicos.ufc.br/resdite/index. Acesso em "dia/mês/ano".

Data de recebimento do artigo: $15 / 10 / 2019$

Data de aprovação do artigo: 19/01/2020

Data de publicação: 1704/2020 